\title{
EFFECT OF FLOW COEFFICIENT AND LOADING COEFFICIENT ON THE RADIAL INFLOW TURBINE IMPELLER GEOMETRY
}

\author{
Samip Shah ${ }^{1}$, Gaurang Chaudhri ${ }^{2}$, Digvijay Kulshreshtha ${ }^{3}$, S. A. Channiwala ${ }^{4}$ \\ ${ }^{1,2,3}$ Assistant Professor, Mechanical Engineering Department, C.K.Pithawalla College of Engineering and Technology, \\ Gujarat, India, ${ }^{4}$ Professor, Mechanical Engineering Department, S. V.National Institute of Technology, Gujarat, India \\ Isamip_mech@yahoo.com, ${ }^{2}$ gaurang_chaudhari2005@yahoo.com, ${ }^{3}$ casanovicdigs@hotmail.com, ${ }^{4}$ sac@med.svnit.ac.in
}

\begin{abstract}
Before attempting to the design of radial inflow turbine, some of the techniques used to describe and present the effect of coefficient on geometry, need to be appreciated. The user of turbine will generally require parameters which readily describe the overall dimension of the machine so that assessments and comparisons can be easily made. The designer requires parameters which will enable him to select the correct machine and make valid comparisons between competing designs. This allows the designer to compute more easily the dimension of the machine at different coefficient, to assess the performance of a range of geometrically similar machines. A paper describes the basic design parameters and effect of coefficient on radial inflow turbine impeller geometry for $25 \mathrm{~kW}$ application.
\end{abstract}

Index Terms: radial turbine design, flow and loading coefficient

\section{INTRODUCTION}

The design of a radial inflow turbine is calculated for different range of flow coefficient and loading coefficient, using turbine design methodology. These are most usefully presented in the form of plots of flow coefficient and loading coefficient.

A number of methods have been suggested for obtaining the overall dimensions of radial gas turbines; the most notable has been that presented by Rohlik[1] in the form of charts related to specific speed and defined slip as the ratio of the tangential velocity at the rotor inlet to the rotor tip speed. Benson[2,3] has analyzed the performance of radial turbines, the methods used in these analysis will be used for the development of the procedure for the prediction of the overall dimensions. Stanitz's [4] formula for the relationship between the slip factor and the number of blades for compressors will be used. The aggregate losses due to friction in the blade passages, clearance effects and disk friction are difficult to predict except on past experience. Benson [5] has made an extensive analysis of a number of radial turbines and has reported on a method for evaluating these losses from test data. The method of analysis developed by Benson to predict the performance characteristics of radial gas turbines is the basis of the design procedure. Baines[6,7] suggested an integrated approach to design a radial inflow turbine.

\section{DESIGN OF RADIAL INFLOW GAS TURBINE IMPELLER FOR 25 kW CAPACITY}

\subsection{Basic Design Concepts for Turbine Rotor}

The rotor is primarily a work transfer device, and any consideration of its design will logically start with Euler turbo machinery equation.

$$
\begin{aligned}
& \frac{W}{m}=U_{2} C_{\theta 2}-U_{3} C_{\theta 3} \\
& =\frac{1}{2}\left[\left(U_{2}^{2}-U_{3}^{2}\right)-\left(C_{w 2}{ }^{2}-C_{w 3}{ }^{2}\right)+\left(C_{2}^{2}-C_{3}{ }^{2}\right)\right]
\end{aligned}
$$

Where subscripts 2 and 3 here denote the rotor inlet and exit planes respectively. Each of three terms in equation makes a contribution to the specific work output. For radial inflow turbine the first term is positive. It also demonstrates why a radial inflow is preferred to a radial outflow machine. For the second term to be positive, CW3 must be greater than CW2, that is to say the rotor should be designed so that the flow relative to the rotor accelerates through the blade passage. The third term is the reverse of the second, and demonstrates the need to maximize the difference between the absolute velocities at inlet $\mathrm{C} 2$ and outlet $\mathrm{C} 3$.

In general the aim is to keep the flow velocities as low a reasonably possible, since many losses including friction and exit kinetic energy increase as the square of velocity. The 
general form of the velocity triangle at the entry and exit of the rotor is to give an increase in relative velocity and a decrease in absolute velocity through the rotor. The inlet blade angle $\beta 2$ of a radial inflow turbine is invariably set to zero at initial stage. Strength limitations normally require that the rotor blades are radially fibered that is to say, the blades are radial in any section taken normal to the rotor axis. This constraints the inlet blade angle to be zero. In order to arrive at a simple evaluation of the optimum incidence angle at the inlet of the runners, the relative flow has been described by the superposition of through flow and relative eddy vortex. In general, however, the optimum incidence of through flow depends solely on the geometric characteristics of the runner, i.e., blade curvature, thickness and solidity. In a well designed turbine operating at its design point, the relative flow angle $\beta 2$ at inlet will match to the rotor so that the flow enters the blade passage with minimum disturbance. At inlet to turbine rotor, the blades are radial. This is a highly stressed region of the rotor, and if they would not be radial, there would be a bending stress in the blades generated by the centrifugal force, which could cause premature damage or failure of the rotor. However, the optimum inlet flow angle is in the region of 100 to -400 . This has been deduced from many experimental measurements of radial turbine performance, and occasionally by direct measurement (Yeo and Baines[11] 1990), and it can also be seen in flow visualizations of the inlet region, such as those shown by Woollley and Hatton[10] (1973) in Figure 1. Here streak lines of the working fluid were visualized by means of entrained particles, and results for three different approach angles are shown in the fig. The most uniform flow distribution was found to be case (b) at an inlet flow angle of -400. The radial inflow condition (a) shows a strong recirculation on the blade suction surface, while the more negative flow angle (c) results in a recirculation on the pressure surface. It would appear from this that the optimum flow condition is probably somewhere between these of cases (a) and (b).
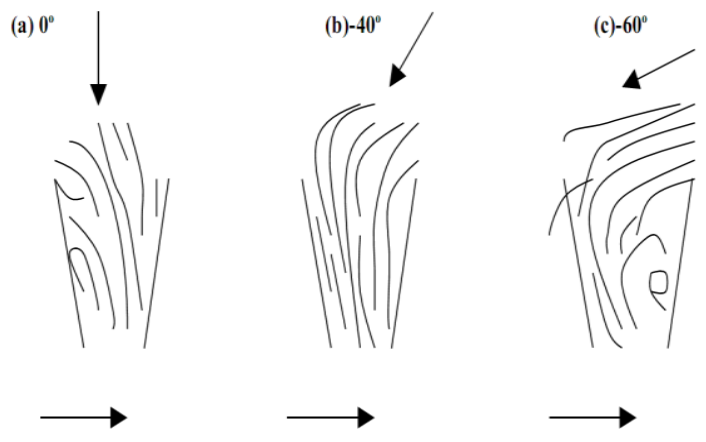

\section{Direction of rotation.}

Figure 1 Conditions for optimum incidence angle [17]

The cause of this behavior is primarily the pressure difference between the pressure and suction surfaces of the rotor blades, which causes the work extraction to occur. This same mechanism causes incidence in axial stages, but now there is an additionally the effect of the decreasing radius, which causes the coriolis acceleration, acting across the passage to diminish as the fluid moves radially inwards. A similar accelerating force at lower radii does not match the strong cross-passage force encountered near blade tips, and as a result a secondary flow is set up in the blade passage in the form of a circulation in the opposite direction to the passage rotation as shown in Figure 1. If the circulation is sufficiently large, the flow will stagnate and separate on the pressure surfaces of the blades. This will happen at large negative incidence. Zero or positive incidence will reduce the strength of the circulation and reduce this tendency, but will have also effect of reducing the cross passage pressure gradient and make the flow more likely to separate on the suction surface. Based on this they gave following calculation. The absolute velocity at exit is normally designed to be axial and small in magnitude. This is because the kinetic energy is lost if the exit flow is not diffused effectively $[7,9]$.

\subsection{Rotor Inlet Design}

The preliminary design presented here is given as a means for determining the outline dimensions of the rotor. The input to the procedure is power, mass flow rate, and rotor speed and expansion ratio. The output is the basic dimensions of the inlet and exit of the rotor, in terms of diameter, blades height and angle. For the given conditions, the mass flow rate was calculated by cycle analysis. The basic assumption and the result of cycle analysis is shown in table 1 and 2 .

Table 1 Assumptions for Cycle Analysis [13]

\begin{tabular}{|l|l|}
\hline Compressor Inlet Pressure & $1.013 \mathrm{bar}$ \\
\hline Compressor inlet temperature & $288 \mathrm{~K}$ \\
\hline Compression ratio & 3 \\
\hline Compressor efficiency & $75 \%$ \\
\hline Compression index of air & 1.4 \\
\hline Specific heat of air & $1.005 \mathrm{~kJ} / \mathrm{Kg} \mathrm{k}$ \\
\hline $\begin{array}{l}\text { Pressure drop in combustion } \\
\text { chamber }\end{array}$ & $6 \%$ \\
\hline Turbine efficiency & $75 \%$ \\
\hline Expansion index of gas & 1.302 \\
\hline Specific heat of gas & $1.315 \mathrm{~kJ} / \mathrm{Kg} \mathrm{k}$ \\
\hline
\end{tabular}

Table 2 Cycle Analysis Results [13]

\begin{tabular}{|l|l|}
\hline Inlet pressure $\mathrm{P}_{01}$ & $2.83 \mathrm{bar}$ \\
\hline Inlet temperature $\mathrm{T}_{01}$ & $1200 \mathrm{~K}$ \\
\hline Outlet static pressure $\mathrm{P}_{3}$ & $1.1 \mathrm{bar}$ \\
\hline Outlet temperature $\mathrm{T}_{03}$ & $1023.75 \mathrm{~K}$ \\
\hline $\mathrm{m}_{\mathrm{g}}$ & $0.1028 \mathrm{Kg} / \mathrm{s}$ \\
\hline
\end{tabular}


The power co efficient is an important parameter. It is given by

$$
S_{w}=\frac{P_{T}}{m^{*} h_{01}}=1-\frac{h_{03}}{h_{01}}=1-\frac{T_{03}}{T_{01}}
$$

Conventional stage loading and flow coefficients which are non dimensional parameters are extensively used in axial turbine design for correlating stage efficiency, but are been used to the same extent in radial turbine design. In fact they are potentially valuable concepts. Unlike axial turbines, in which the blade speed is nearly constant through a blade row, in radial turbines there is a wide variation in blade speed due to the radius change between inlet and exit, and therefore choice of blade speed is used to define these coefficients is arbitrary. The stage loading coefficient here is based on the inlet blade speed U2, and can be expressed using the Euler turbo machinery equation as

$\psi=\frac{\Delta h_{0}}{U_{2}^{2}}=\frac{C_{\theta 2}}{U_{2}}-r \frac{C_{\theta 3}}{U_{2}}$

Where, $\quad r=\frac{r_{3 S}}{r_{2}}$ is the rotor radius ratio. The exit swirl is normally fairly small, so that the second term on the right hand side can be neglected from which U2 can be found out. The flow coefficient is defined in terms of the exit meridional velocity, also non- dimensionalized by the inlet blade speed,

$\phi=\frac{C_{m 2}}{U_{2}}$ 5

Figure 2 shows a correlation of radial turbine efficiency with these coefficients using test data taken from about forty different stages and a variety of sources. The reported total to static efficiency is shown in the figure proves to be sufficiently good to allow lines of constant efficiency to be drawn over a wide area of the map. The data used here comes from a wide variety of stage designs, but this only serves to emphasize the universality of the chart.

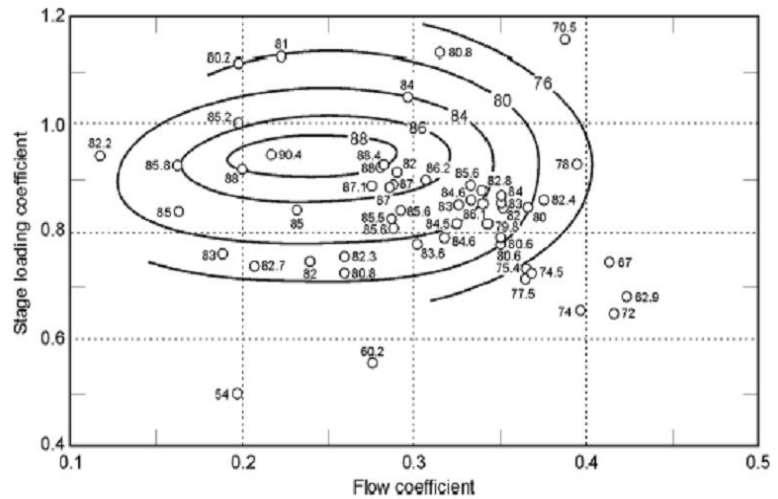

Figure 2 Correlation of measured efficiency of a range of turbine designs with stage loading and flow coefficients (Chen and Baines) [12]

Maximum efficiency occurs for flow coefficients in the range $0.2-0.3$, and at loading coefficients between about 0.7 and 0.9 . In many cases designers have used values significantly different from these optima [12].

The design procedure begins by choosing suitable values of the loading and flow coefficients, using figure 2 as a guide. The design parameters then can be calculated as follows.

$C_{m 2}=\phi^{*} U_{2}$

6

$C_{2}=\sqrt{C_{\theta 2}^{2}+C_{m 2}^{2}}$

Taking consideration of the blade inlet flow angle (as described by Figure 1) it can be shown as in Figure 3. At the rotor inlet it is necessary to establish the magnitude and direction of the absolute velocity vector so that an appropriate volute can be designed to generate the requirements. Establishment of the direction of the relative velocity will lead to the design of the rotor inlet blade angle which will accept this vector with minimum of incidence loss.

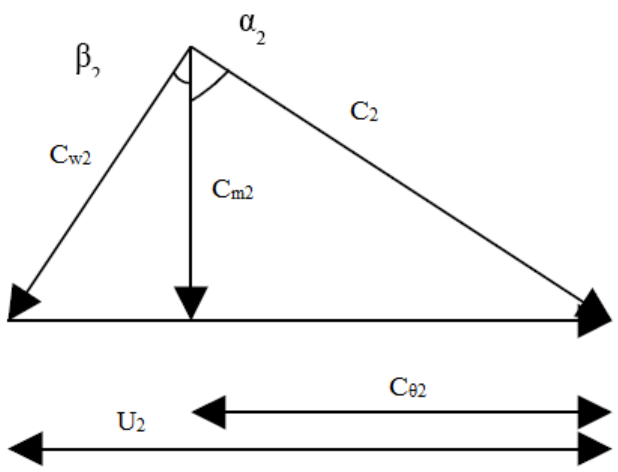

Figure 3 Velocity triangles at inlet [9] 
The other parameters like absolute flow angle, relative velocity, flow angle and Mach number at impeller inlet can be calculate as

$$
\begin{aligned}
& \alpha_{2}=\tan ^{-1} \frac{C_{\theta 2}}{C_{m 2}} \\
& C_{w 2}=\sqrt{C_{m 2}^{2}+\left(U_{2}-C_{\theta 2}\right)^{2}} \\
& \beta_{2}=\tan ^{-1} \frac{U_{2}-C_{\theta 2}}{C_{m 2}} \\
& M_{u}=\frac{U_{2}}{a_{01}}
\end{aligned}
$$

The static temperature and pressure at the inlet to the rotor are $T_{2}=T_{02}-\left(\frac{C_{2}^{2}}{2 * C_{P}}\right)$

$P_{2}=P_{02}\left(\frac{T_{2}}{T_{02}}\right)^{\frac{n-1}{n}}$

Where $\mathrm{T} 02=\mathrm{T} 01$ and $\mathrm{P} 02=\mathrm{P} 01-\Delta \mathrm{P} 0$. Here $\Delta \mathrm{P} 0$ is the total pressure loss in the stator. The stator loss is usually only a small fraction of the overall turbine loss, it may be justifiable to ignore it and treat the stator as isentropic.

The inlet area follows

$A_{2}=2 * \pi * r_{2} * b_{2}$

Applying continuity equation

$m=C_{m 2} * A_{2} * \frac{p_{2}}{R * T_{2}}$

\subsection{Rotor Discharge Condition}

From the rotor discharge conditions the designer must establish the relative flow angle, from which the blade angle can be derived, and exducer radius ratio r3S/r2. An assumption of swirl at the discharge will be made. In practice this may not occur over the full length of the blade, at any operating condition, due to the secondary flows set up in the rotor passage. Nonetheless the assumption of zero swirl at the design condition is probably the best that can be made, unless a deliberate decision is made for a specific degree of swirl which will also be difficult to achieve in practice.

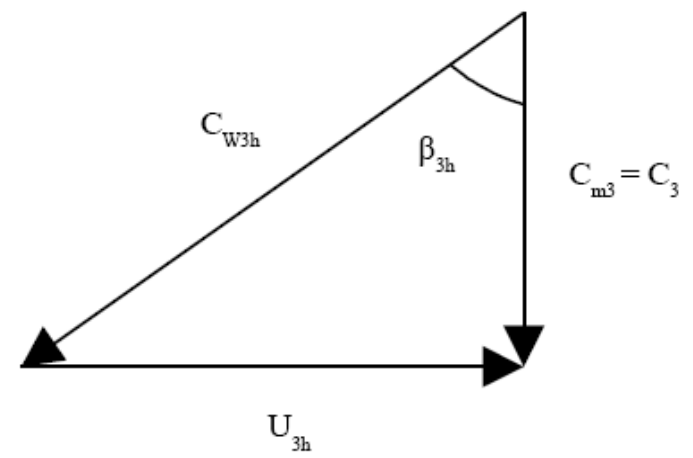

(a).Velocity triangle for hub

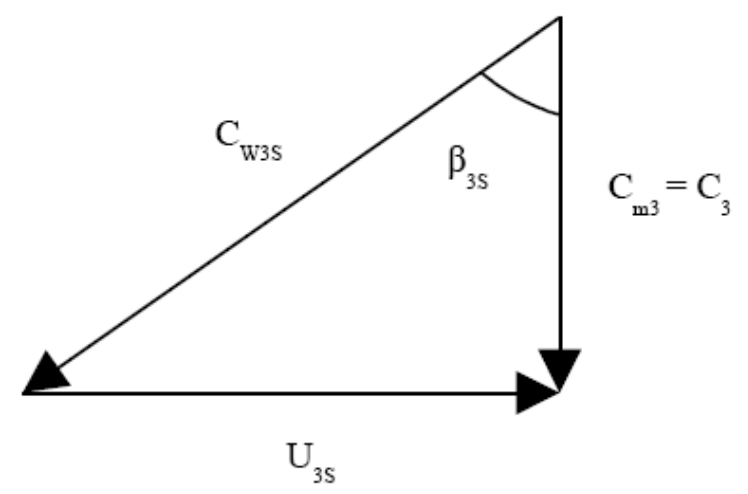

(b) Velocity triangle for shroud

Figure 4 Velocity triangles at exit [9]

The rotor meridional velocity ratio $\xi=\frac{C_{m 2}}{C_{m 3}}$

For the case of minimum inlet Mach number, this ratio is given by

$$
\frac{r_{3 s}}{r_{2}}=W_{R} * \sin \beta_{3} * \sqrt{\frac{1-\cos \beta_{2}}{1+\cos \beta_{2}}}
$$

The relative velocity ratio WR In this case the radius ratio is a linear function of the relative velocity for any defined inlet and exit relative flow angles. In above equation WR is the relative velocity ratio $\mathrm{CW} 3 / \mathrm{CW} 2$ Rohlik [1] assumed a value of two, which has some justification since a value substantially in excess of unity is required to ensure a good expansion through the rotor. Also Rohlik suggested that the relative Mach number is a minimum when the relative flow angle $(\beta 3)$ is about -55 degrees.

Now, The relative mach number at shroud exit 


$$
M_{R 3 s}=\frac{C_{w 3 s}}{\sqrt{n * R^{*} T_{3}}}
$$

Also exit static temperatures is

$$
T_{3}=T_{03}-\left(\frac{C_{3}^{2}}{2 * C_{P}}\right)
$$

Now the flow at the exit is axial. So $\mathrm{C} 3=\mathrm{Cm} 3$.

For isentropic condition

$P_{3}=P_{03} *\left(\frac{T_{3}}{T_{03}}\right)^{\frac{n}{n-1}}$

According to Glassman [14] as shown the number of blade and solidity can be given by

$Z_{B}=\frac{\pi *\left(110-\alpha_{2}\right) * \tan \alpha_{2}}{30}$

Solidity is defined as

$$
L=\frac{s d_{2}}{Z_{B}}
$$

Above equation has been solved for the different range of flow coefficient and loading coefficient found in literature.

\section{Results and Discussion}

The graphs have been plotted for Impeller inlet diameter, Impeller inlet width, Impeller shroud radius, Impeller hub radius, Impeller axial length and No of Impeller blade vs. Impeller speed for the different values of flow coefficient and loading coefficient.

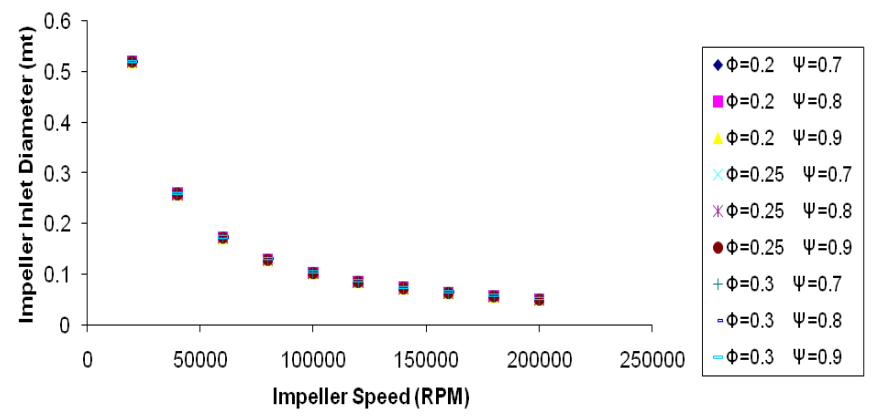

Figure 5 Impeller Inlet Diameter vs Impeller speed

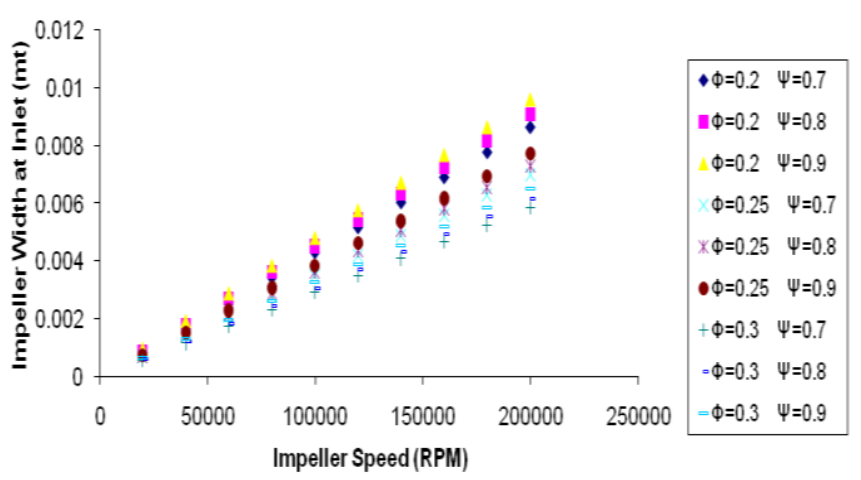

Figure 6 Impeller Inlet Width vs Impeller Speed

The figure 5 shows that there is no change in inlet diameter by changing the value of flow coefficient and blade loading coefficient. The impeller inlet diameter is only varying by changing the impeller speed. The impeller inlet diameter decreases as impeller speed increases. The figure 6 shows that as the impeller speed increases the impeller inlet width increases. Also it can be seen that impeller inlet width varies by changing the flow coefficient and loading coefficient. As the value of flow coefficient increases inlet width decreases because the value of mean velocity proportional to the flow coefficient. In equation 15 the mass flow rate, static pressure and static temperature at inlet are fixed, only variables are mean velocity and impeller inlet area. So, as the mean velocity increases the inlet area decreases which leads to decrease inlet width. By increasing the value of loading coefficient the inlet width also increases because by increasing the value of loading coefficient the value of whirl component is increases, that increase the absolute velocity and decrease the static pressure which ultimately increase the inlet area so that the inlet width of impeller.

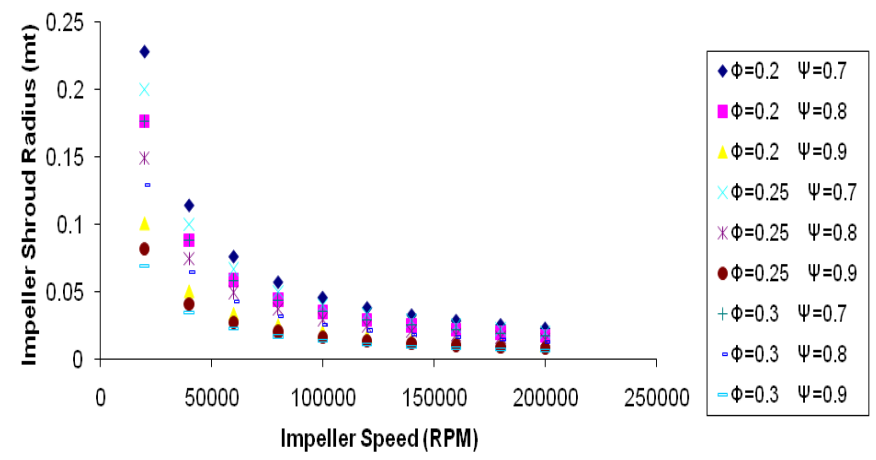

Figure 7 Impeller Shroud Radius vs Impeller Speed 


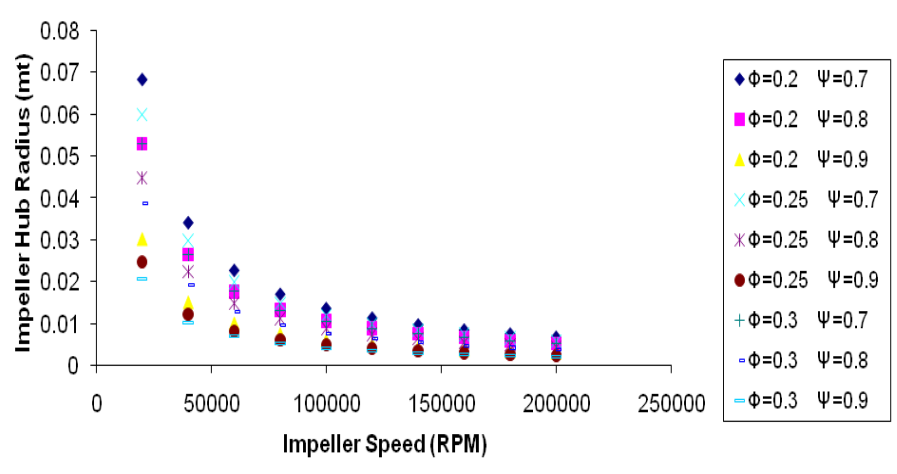

Figure 8 Impeller Hub Radius vs Impeller Speed

The figure 7 shows that by increasing the value of flow coefficient and loading coefficient the value of shroud radius decreases. This is because the value of impeller shroud radius is proportional to inlet flow angle i.e. $\beta 2$ (equation 16). As the value of flow coefficient and loding coefficient increases the value of $\beta 2$ decreases which leads to decrease the impeller shroud radius.

By increasing the value of flow coefficient and loading coefficient the value of hub radius decreases as the hub radius is directly proportional to the impeller shroud radius (Figure 8).

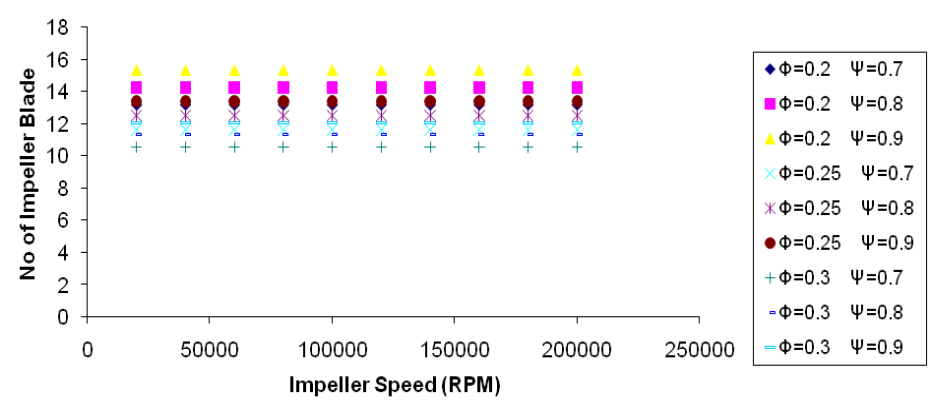

Figure 9 Number of Impeller Blade vs Impeller Speed

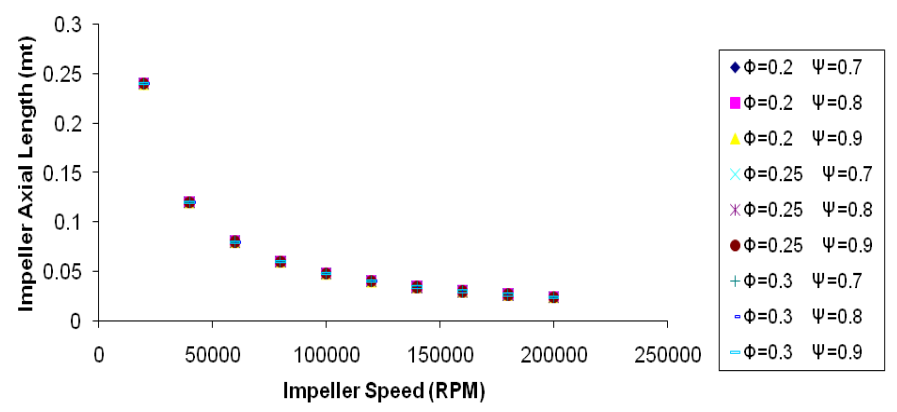

Figure 10 Impeller Axial Length vs Impeller Speed
The figure 9 shows that there is no change in number of blades by changing the impeller speed but by increasing the value of loading coefficient the number of blade increases and by increasing the value of flow coefficient the number of blade decreases. The figure 10 shows that there is no chnage in axial length by changine the value of flow coefficient and blade loading coefficient. The impeller axial length is only varying by changing the impeller speed. The axial length is decreases as the impeller speed increases as it is directly proportional to the impeller inlet diameter.

\section{CONCLUSIONS}

The effect of flow coefficient, loading coefficient and impeller speed on the overall dimensions of radial turbine impeller has been studied in the present paper and the salient feature of the results obtained are concluded as follows:

1. Impeller inlet diameter will not change by changing the value of flow coefficient and loading coefficient, but it decreases by increasing the impeller speed.

2. Impeller inlet width decreases by increasing the value of coefficient and it increases by increasing the value of loading coefficient and impeller speed.

3. Impeller shroud radius and Impeller hub radius decreases by increasing the value of coefficient, loading coefficient and impeller speed.

4. Number of impeller blades decreases by increasing the value of coefficient, increases by increasing the value of loading coefficient and unchanged by increasing the value of impeller speed.

5. Axial length of impeller remained unchanged by increasing the value of coefficient and loading coefficient, but decreases by increasing the impeller speed.

\section{NOMENCLATURE}

$\begin{array}{ll}\text { A } & \text { Area } \\ \text { a } & \text { Speed of sound } \\ \text { b } & \text { Blade width } \\ \text { C } & \text { absolute velocity } \\ \text { Cp } & \text { Specific heat at constant pressure } \\ \mathrm{Cs} & \text { Isentropic velocity (Spouting velocity) } \\ \mathrm{C}_{\mathrm{V}} & \text { Specific heat at constant volume } \\ \mathrm{d} & \text { Diameter of rotor } \\ \mathrm{h} & \text { enthalpy } \\ \mathrm{L} & \text { Passage length } \\ \mathrm{M} & \text { Mach number } \\ \mathrm{m} & \text { mass flow rate } \\ \mathrm{N} & \text { Speed of rotation } \\ \mathrm{P} & \text { Pressure } \\ \mathrm{R}_{\mathrm{g}} & \text { Gas constant } \\ \mathrm{Re} & \text { Reynolds number } \\ \mathrm{r} & \text { Rotor radius } \\ \mathrm{r}_{3 \mathrm{~S}} / \mathrm{r}_{2} & \text { Radius ratio } \\ \mathrm{S}_{\mathrm{W}} & \text { Non dimensional power ratio } \\ \mathrm{S} & \text { Solidity, Swirl co efficient }\end{array}$


T Temperature

$\mathrm{U} \quad$ Blade speed

$\mathrm{C}_{\mathrm{W}} \quad$ Relative velocity

$\mathrm{W}_{\mathrm{R}} \quad$ Relative velocity ratio

$\mathrm{Z} \quad$ Axial length

$\mathrm{Z}_{\mathrm{B}} \quad$ Number of blades

$\alpha \quad$ Absolute flow angle

$\beta \quad$ Relative flow angle

n Specific heat ratio

$\eta \quad$ Efficiency

$\rho \quad$ Density

$\varphi \quad$ Flow coefficient

$\psi \quad$ Blade loading coefficient

$\xi \quad$ Meridional velocity ratio

$\varepsilon_{r} \quad$ Exducer hub to shroud ratio

\section{SUBSCRIPTS}

0 Stagnation, Stator inlet

1 Turbine inlet

2 Rotor inlet

3 Rotor exit

\section{REFERENCES}

[1] H. E. Rohlik, "Analytical determination of radial inflow turbine design geometry for maximum efficiency". NASA TN 4384 (1968).

[2] R. S. Benson, "An analysis of the losses in a radial gas turbine”, Proc. L Mech. E. IN, (Part 3J), 41-53 (1965-66).

[3] R. S. Benson, W. G. Cartwright and S. K. Das, "An analysis of the losses in the rotor of a radial flow gas turbine at zero incidence". Proc. L Mech. E. 182, (Part 3H), (1967--68).

[4] J. D. Stanitz and G. O. Ellis, "Two-dimensional compressible flow in centrifugal compressors with straight blades". NACA Report No. 954.

[5] R. S. Benson, "Prediction of performance of radial gas turbines in automotive turbochargers". A, S.M.E. Gas Turbine Conference, Houston, Texas. Paper No. 71-Gt66 (28 Mar.-I Apr. 1971).

[6] Nick Baines, "Radial turbines: an integrated design approach" Concepts NREC Chipping Norton, UK.

[7] N.C. Baines and A. Whitfield, "Design of radial turbomachines". Longman Scientific \& Technical, John Wiley \& Sons, NY.

[8] Mehul P. Pandya, Dr. S. A. Channiwala 2004, "Design, Optimization And Simulation Of Radial Inflow Gas Turbine In Small Capacity Range", deprtement of mechanical engg., SVNIT.

[9] Hany Moustapha, N.C.Baines, David Japiske 2003, "Axial and Radial Turbines", Concepts , NREC

[10] Wooley N.H., Hatton 1973, " Viscous flow in radial turbo machines blade passages". Inst Mach Engineers Conference proceeding, 1973-3.
[11] Yeo J. H.,Baines N.C. 1990, “ Pulsating flow behavior in twin entry vane less radial inflow turbine in turbo charging and turbo chargers", Inst of Mechanical Engineers, 113-22.

[12] Chain H.,Baines N.C.,1992, “ Performance parameters and assessment", Lecture series, V.K.I., U.K.

[13] Mehta Sumit S, Shah Bhavin, Singh B., Trivedi H., "Design of Radial Inflow Turbine for Micro Gas Turbine Applications",Project Report, C.K.Pithawalla College of Engg. and Tech., 2009.

[14] Glassman A.J. 1976, "Design and analysis of radial gas turbines". NASA-TN 8164. 